\title{
ON FUNCTIONS IN THE BALL ALGEBRA
}

\author{
P. WOJTASZCZYK
}

\begin{abstract}
We show that there exists a function in a ball algebra such that almost every slice function has a series of Taylor coefficients divergent with every power $p<2$.
\end{abstract}

In §7.2 of [3] W. Rudin gives some examples of boundary behavior of holomorphic functions in complex balls of dimension 2 and 3. It was observed in [4, Remark 1.10], that using Theorem 1.2 of [4] such examples can be constructed in arbitrary dimension. In the present note we further pursue this idea. It is well known that in one variable there exists a function $f(z)=\sum_{n=0}^{\infty} a_{n} z^{n}$ analytic for $|z| \leq 1$ such that $\sum\left|a_{n}\right|^{p}=\infty$ for all $p<2$. Our theorem generalizes this fact to functions of several variables.

In this note, $B$ will always denote the unit ball in the complex $d$-dimensional space $C^{d}, S$ will stand for the unit sphere and $\sigma$ for the rotation invariant probability measure on $S . A(B)$ will denote the ball algebra of all functions analytic in $B$ and continuous in $\bar{B}$. For $0<p \leq \infty,\|f\|_{p}$ denotes $\left(\int_{S}|f(\zeta)|^{p} d \sigma(\varsigma)\right)^{1 / p}$. If $f$ is a holomorphic function in $B$ then it has a unique homogeneous expansion as $f=$ $\sum_{n=0}^{\infty} f_{n}, f_{n}$ is an analytic polynomial homogeneous of degree $n$.

It was shown in [4, Theorem 1.2], that there exist polynomials $\left(p_{n}\right)$ homogeneous of degree $n$ on $B$ such that

$$
\left\|p_{n}\right\|=1 \text { and }\left\|p_{n}\right\|_{\infty} \leq \chi .
$$

( $\chi$ depends only on the dimension of the ball, it can be taken $\chi=2^{d} / \sqrt{\pi}$.) Those polynomials will be crucial in our further considerations. In particular, we will use the following inequality (cf. [4, Proposition 1.6])

$$
\left\|\sum_{k=0}^{\infty} \alpha_{n} p_{2^{n}}\right\|_{p} \sim\left(\sum_{k=0}^{\infty}\left|\alpha_{n}\right|^{2}\right)^{1 / 2}, \quad 0<p<\infty .
$$

We will also use the fact that Cauchy Integral $C[\mu]$ maps continuously measures on $S$ into all $H_{p}(B), p<1$ (cf. $[3,6.2]$ ). $B$.

The book [3] is an excellent source of information about the function theory in

Proposition. The operator $T: A(B) \rightarrow \ell_{2}$ defined by $T(f)=\left(\left\langle f, p_{2^{n}}\right\rangle\right)_{n=0}^{\infty}$ is a surjective map.

ProOF. This map is clearly continuous. By duality it is enough to show that $T^{*}: \ell_{2} \rightarrow A(B)^{*}$ is an isomorphic embedding i.e. $\left\|T^{*}\left(\alpha_{n}\right)\right\|_{A(B)^{*}} \geq C\left(\sum\left|\alpha_{n}\right|^{2}\right)^{1 / 2}$.

Received by the editors July 21, 1981.

1980 Mathematics Subject Classification. Primary 32A40; Secondary 32A05, 32E25.

(4) 1982 American Methematical 8ociety 0002-9939/81/0000-1074/801.75 
One easily checks that $T^{*}\left(\alpha_{n}\right)(f)=\left\langle f, \sum_{n=0}^{\infty} \alpha_{n} p_{2^{n}}\right\rangle$. Let $\mu$ be a measure on $S$ such that, for $f \in A(B), T^{*}\left(\alpha_{n}\right)(f)=\int f d \mu$ and $\|\mu\|=\left\|T^{*}\left(\alpha_{n}\right)\right\|$. Then obviously $C[\bar{\mu}]$, the Cauchy Integral of $\bar{\mu}$, equals $\sum_{n=0}^{\infty} \alpha_{n} p_{2^{n}}$, so (by properties of $C[\cdot]$ and $(* *))$

$\left\|T^{*}\left(\alpha_{n}\right)\right\|=\|\mu\|=\|\bar{\mu}\| \geq C\|C[\bar{\mu}]\|_{1 / 2}=C\left\|\sum_{n=0}^{\infty} \alpha_{n} p_{2^{n}}\right\|_{1 / 2} \geq C\left(\sum_{n=0}^{\infty}\left|\alpha_{n}\right|^{2}\right)^{1 / 2}$.

COROLLARY. There exists a constant $C$ such that for every sequence $\left(\alpha_{n}\right)_{n=0}^{\infty}$ with $\alpha_{n}=0$ for $n \leq K$ and $n \geq N$ (for some integers $K$ and $N$ ) there exists $f \in A(B)$ such that

$$
\|f\|_{\infty} \leq C\left(\sum_{n=0}^{\infty}\left|\alpha_{n}\right|^{2}\right)^{1 / 2}, \quad T f=\left(\alpha_{n}\right)
$$

and the homogeneous expansion of $f$ has a form

$$
\sum_{s=2^{K}+1}^{2^{N}-1} f_{s}
$$

ProOF. Let $\beta$ be a piecewise linear function given by

$$
\beta(t)= \begin{cases}0 & \text { if } t \leq 2^{K} \text { or } t \geq 2^{N} \\ 1 & \text { if } 2^{K+1} \leq t \leq 2^{N-1} \\ \text { linear } & \text { otherwise. }\end{cases}
$$

It follows from the classical de la Vallee-Poussin theorem that the map $f=$ $\sum_{n=0}^{\infty} f_{n} \mapsto \sum_{n=0}^{\infty} \beta(n) \cdot f_{n}$ maps $A(B)$ continuously into $A(B)$, and has norm bounded by an absolute constant. This observation and the proposition proves the corollary.

THEOREM. There exists a function $f \in A(B)$ with the homogeneous expansion $f=\sum_{n=0}^{\infty} f_{n}$ such that for every $p<2$ and $\sigma$-almost all $\varsigma \in S, \sum_{n=0}^{\infty}\left|f_{n}(\zeta)\right|^{p}=\infty$ i.e., almost every slice function of $f$ has a series of Taylor coefficients divergent with every power $<2$.

PROOF. Let us denote $2^{2^{r+k}}$ by $\gamma(r, k)$. Repeated use of the corollary gives a sequence of functions $g^{r} \in A(B)$ such that

(1) the homogeneous expansion of $g^{r}$ has form

$$
g^{r}=\sum_{\gamma(r, 0)+1}^{\gamma(r+1,0)-1} g_{k}^{r}
$$

(2) $\left\|g^{r}\right\|_{\infty} \leq C$;

(3) $\left\langle g^{r}, p_{\gamma(r, k)}\right\rangle=2^{-r / 2}, k=1,2, \ldots, 2^{r}-1$.

The properties (1)-(3) imply that there exist two constants $\alpha, \beta$ such that for every $r$ and $p$

(4) $\sum_{k}\left|g_{k}^{r}(\zeta)\right|^{p} \geq \alpha 2^{r(1-p / 2)}$ on a set $A_{r} \subset S$ with $\sigma\left(A_{r}\right) \geq \beta$.

ProOF OF (4). By (3) and (*) we infer 
(5) $\int\left|g_{\gamma(r, k)}^{r}\right| d \sigma \geq \chi^{-1} 2^{-r / 2}$.

Since $C^{2} \geq\left\|g^{r}\right\|_{2}^{2}>\sum_{k=1}^{2^{r}-1}\left\|g_{\gamma(r, k)}^{r}\right\|_{2}^{2}$ we see that for at least $2^{r-1}$ of the functions $\left(g_{\gamma(r, k)}^{r}\right)_{k=1}^{2^{r}=1}$ we have

(6) $\left\|g_{\gamma(r, k)}^{r}\right\|_{2} \leq 2 C 2^{-r / 2}$.

Using (5) for each $k$ satisfying (6) we obtain a set $B_{k}, \sigma\left(B_{k}\right) \geq 16^{-1} C^{-2} \chi^{-2}$, such that $\left|g_{\gamma(r, k)}^{r}(\zeta)\right| \geq 1 / 2 \chi \cdot 2^{-r / 2}$ for $\zeta \in B_{k}$.

From this follows that for some $\alpha$ and $\beta$ (independent of $r$ ) the set $A_{r}$ of all points in $S$, which belong to at least $\alpha \cdot 2^{r}$ sets $B_{k}$, has measure $\geq \beta$. This clearly proves (4).

Using the "scrambling lemma" [3, p. 129] we find a sequence $U_{r}$ of unitary maps such that almost every $\zeta \in S$ belongs to infinitely many sets $U_{r}\left(A_{r}\right)$. We define the desired function by

(7) $f=\sum_{r=1}^{\infty} r^{-2} g^{r} \circ U_{r}^{-1}$.

By (1) we infer that the homogeneous expansion $\sum_{n=0}^{\infty} f_{n}$ for $f$ actually equals $\sum_{r=1}^{\infty} \sum_{k=\gamma(r, 0)+1}^{r(r+1,0)} r^{-2} g_{k}^{r} \circ U_{r}^{-1}$. Since $\left\|g^{r} \circ U_{r}^{-1}\right\|_{\infty}=\left\|g_{r}\right\|_{\infty} \leq C$ the series (7) is absolutely convergent and defines a function in $A(B)$. By (4), for $\varsigma \in U_{r}\left(A_{r}\right)$, we have $\sum_{k}\left|g_{k}^{r} \circ U_{r}^{-1}(\zeta)\right|^{p} \geq \alpha 2^{r(1-p / 2)}$

Given $\zeta \in S$

$$
\sum_{n=0}^{\infty}\left|f_{n}(\zeta)\right|^{p}=\sum_{r=1}^{\infty} r^{-2} \sum_{k=\gamma(r, 0)+1}^{r(r+1,0)-1}\left|g_{k}^{r} \circ U_{r}^{-1}(\zeta)\right|^{p} \geq \sum_{r: \zeta \in U_{r}\left(A_{r}\right)} \alpha r^{-2} 2^{r(1-p / 2)}
$$

This sum is clearly infinite for every $p<2$ and every $\zeta$ belonging to infinitely many sets $U_{r}\left(A_{r}\right)$. This completes the proof.

REMARK 1. I do not know the answer to the following question: given $\left(\alpha_{n}\right) \in \ell_{2}$ does there exist $f=\sum f_{k} \in A(B)$ such that $f_{2^{k}}=\alpha_{k} p_{2^{k}}$ ? A positive answer to this question would imply the theorem.

REMARK 2. Our proof of the proposition is the same as the one given for the disc algebra in [2, Proposition 3.1]. In one variable there are also beautiful constructive proofs (e.g. in [1]). It would be interesting to have such a proof in our case.

\section{REFERENCES}

1. J. Fournier, An interpolation problem for coefficients of $H_{\infty}$ functions, Proc. Amer. Math. Soc. 42 (1972), 402-408.

2. A. Pełczynski, Banach spaces of analytic functions and absolutely summing operators, CBMS Regional Conference Series No. 30, Amer. Math. Soc., Providence, R. I. 1977.

3. W. Rudin, Function theory in the unit ball of $C^{n}$, Springer-Verlag, New York, 1980.

4. J. Ryll and P. Wojtaszczyk, On homogeneous polynomials on a complex ball, Trans. Amer. Math. Soc. (to appear).

Department of MAThematics, The University of TeXas, Austin, TeXas 78712 Institute of Mathematics, Polish ACAdemy of Sciences, 00-950 WarsaW, POLAND 